Original Research Paper

\title{
Change in Temperature of Skin, Blood, Muscle and Bone Tissues Due to Mobile Phone Radiations at Frequencies 800, 900, 1800 and $2450 \mathrm{MHz}$
}

\author{
Mushtaq Ahmed Bhat and Vijay Kumar \\ Department of Physics, Graphic Era University Dehradun, India
}

Article history

Received: 03-05-2015

Revised: 11-05-2015

Accepted: 01-06-2015

Corresponding Author:

Mushtaq Ahmed Bhat

Department of Physics, Graphic

Era University Dehradun, India

Email:dmbhat5979@gmail.com

\begin{abstract}
The mathematical calculation of change in temperature of skin, blood, muscle and bone tissues of human being working at the frequency of $800,900,1800$ and $2450 \mathrm{MHz}$ is done in this manuscript. The power of the antenna of mobile phone is of $2 \mathrm{~W}$. The calculation is made at the various depths, by taking the different distances of human body from the mobile phone. The depth of penetration is ranging from 0.1 to $0.5 \mathrm{~mm}$ inside the body tissues. The thermoregulation system of the body can maintain the body temperature but at some extent. Variation of change in temperature due to the exposure of $15,30,45$ and $60 \mathrm{~min}$ in selected tissues is also analysed.
\end{abstract}

Keywords: Temperature Change, Induced Electric Field, Thermoregulatory Mechanism, Mobile Phone Handset and Electromagnetic Waves

\section{Introduction}

Electromagnetic fields are all around due to mobile phone and mobile phone tower radiations keep our heart beating, brain thinking and muscles moving they are even within our bodies. During speaking, human being absorb some electromagnetic energy when it is exposed to it. This interaction of electromagnetic energy with the body can cause both thermal and non-thermal effects (Kumar et al., 2008).

Depending on the frequency of electromagnetic radiation, the human body interacts with such a field via induced currents and thermal effects. The field generated within the body, so called thermal field is determined by the amount by which a body is influenced by electromagnetic fields (Gandhi and Mohammad, 2008).

The absorbed mobile phone radiations produced molecular vibration and convert the energy into heat. When the rate of energy absorption is high it produces heating effects in the living tissues (Ozen et al., 2009). The biological effects of radiofrequency energy depend on the rate at which power is absorbed (Osepchuk and Petersen, 2003). If the organism cannot dissipate this heat energy as fast as then the internal temperature of the body will rise. Thus the thermal interaction may cause tissue heating by deposition of power from ionic conductivity of the tissue as well as losses associated with motion of the molecules caused by time vibration of electromagnetic field (Furse et al., 2009). Exposure to very high radiofrequency power densities can result increase in temperature heat human body tissues. During the expose of high radio frequency levels tissue damage will occur. Under certain conditions exposure to At power density levels of 1 to $10 \mathrm{~mW} / \mathrm{cm}^{2}$ the radio frequency energy can result in heating of body, testis and eyes are to particularly known to heating by radio frequency energy because available of blood flow to dissipate the excessive heat energy. Such change in sperm mobility effects causes the temporary sterility after the exposure testis to high radio frequency radiation level. Most of the experimental investigations rabbits and mice have been employed for biological effects of radio frequency exposure (James, 2003; Karunarathna and Dayawansa, 2006).

One well-understood the effect of mobile phone wave is dielectric heating in which the living tissues of human being are heated by the rotations of polar molecules. Mobile phone radiation penetrates in tissues to a distance proportional to their power density. That is why it is dangerous to live close to high power mobile phone tower for a long time.

\section{Method and Calculation}

When human body is exposed to the $E M$ wave of electric field $E_{r m s}$, it penetrates into the body. It results into inside or induced field $E_{i}$ at a given depth $Z$ given by Equation 1: 


$$
E_{i}=E_{r m s} e^{(-z / \delta)}
$$

where, $\delta$ is skin depth.

As we want to access the effect of mobile phone tower whose power is the only parameter we know, we have to relate the electric field (more generally $E_{r m s}$ ) to the power of transmission Equation 2:

$$
\begin{aligned}
& E_{r m s}=10.946 / r(\text { for handset }) \text { and } \\
& E_{r m s}=34.641 / r(\text { for tower })
\end{aligned}
$$

where, $r$ is the radius of spherical wave front around mobile phone handset and their tower.

The Specific Absorption Rate (SAR) can be defined as Equation 3:

$$
S A R=\frac{\sigma E_{i}^{2}}{\rho}
$$

where, $E_{i}$ is the field inside that material, $\sigma$ is the conductivity of the biological material, $\rho$ is the density of bio material Equation 4:

$$
C \times \Delta T=\operatorname{SAR}(\Delta t)
$$

where, $\Delta t$ is time in seconds, $C$ is specific heat of biomaterial.

The dielectric properties of the body tissues are taken from Gabriel et al. (1996a; 1996b; 1996c) for the calculation of change in temperature of different tissues. The change in temperature 15, 30, 45 and $60 \mathrm{~min}$ for mobile phone in consequence of specific absorption rate is given in the following tables respectively. The depth inside the tissues is taken $0.1,0.2,0.3,0.4$ and $0.5 \mathrm{~mm}$ for the calculation of change in temperature at different distances from the mobile phone working at the frequencies of $800,900,1800$ and $2450 \mathrm{MHz}$. The power of mobile phone is taken as $2 \mathrm{~W}$.

\section{Standard Values}

- $\quad \mathrm{z}=0.1,0.2,0.3,0.4$ and $0.5 \mathrm{~mm}$

- At $800 \mathrm{MHz}, \sigma=0.80864 \mathrm{~W} \mathrm{~K}^{-1} \mathrm{~m}^{-1}$, skin depth $\delta$ $=45.59 \mathrm{~mm}$

- At $900 \mathrm{MHz}, \sigma=0.84465 \mathrm{~W} \mathrm{~K}^{-1} \mathrm{~m}^{-1}$, of skin depth $\delta=43.352 \mathrm{~mm}$

- At $1800 \mathrm{MHz}, \sigma=1.232 \mathrm{~W} \mathrm{~K}^{-1} \mathrm{~m}^{-1}$, skin depth $\delta=$ $28.808 \mathrm{~mm}$

- At $2450 \mathrm{MHz}, \sigma=1.5919 \mathrm{~W} \mathrm{~K}^{-1} \mathrm{~m}^{-1}$, skin depth $\delta$ $=28.808 \mathrm{~mm}$

The value of density $\rho$ for skin $=1070 \mathrm{~kg} \mathrm{~m}^{-3}$, for blood $=1060 \mathrm{~kg} \mathrm{~m}^{-3}$, for muscles $=1050 \mathrm{kgm}^{-3}$, for bone $=1520 \mathrm{~kg} \mathrm{~m}^{-3}$

Specific heat $\mathrm{C}$ for skin $=3662 \mathrm{~J} \mathrm{~K}^{-1} \mathrm{~kg}^{-1}$, blood $=$ $4186 \mathrm{~J} \mathrm{~K}^{-1} \mathrm{~kg}^{-1}$, muscles $=3639 \mathrm{~J} \mathrm{~K}^{-1} \mathrm{~kg}^{-1}$ and bone $=$ $1590 \mathrm{~J} \mathrm{~K}^{-1} \mathrm{~kg}^{-1}$.

\section{Results and Discussion}

In most of the countries in the world 800, 900, 1800 and $2450 \mathrm{MHz}$ frequency of electromagnetic waves are used for communication. In this chapter, we discuss about four frequencies of mobile phone waves. Calculation of change in temperature for skin, blood, muscles and bones at different depth and different distances are made. The penetrated electromagnetic wave absorbed inside the human body tissues and deposited in the form of extra energy. This energy increases the temperature of tissues which are previously at thermostatic state.

When a mobile phone handset move from 15 to 1 $\mathrm{cm}$ distance towards person, then the temperature of tissue increases. The results are represented by Table 17 and Fig. 1.

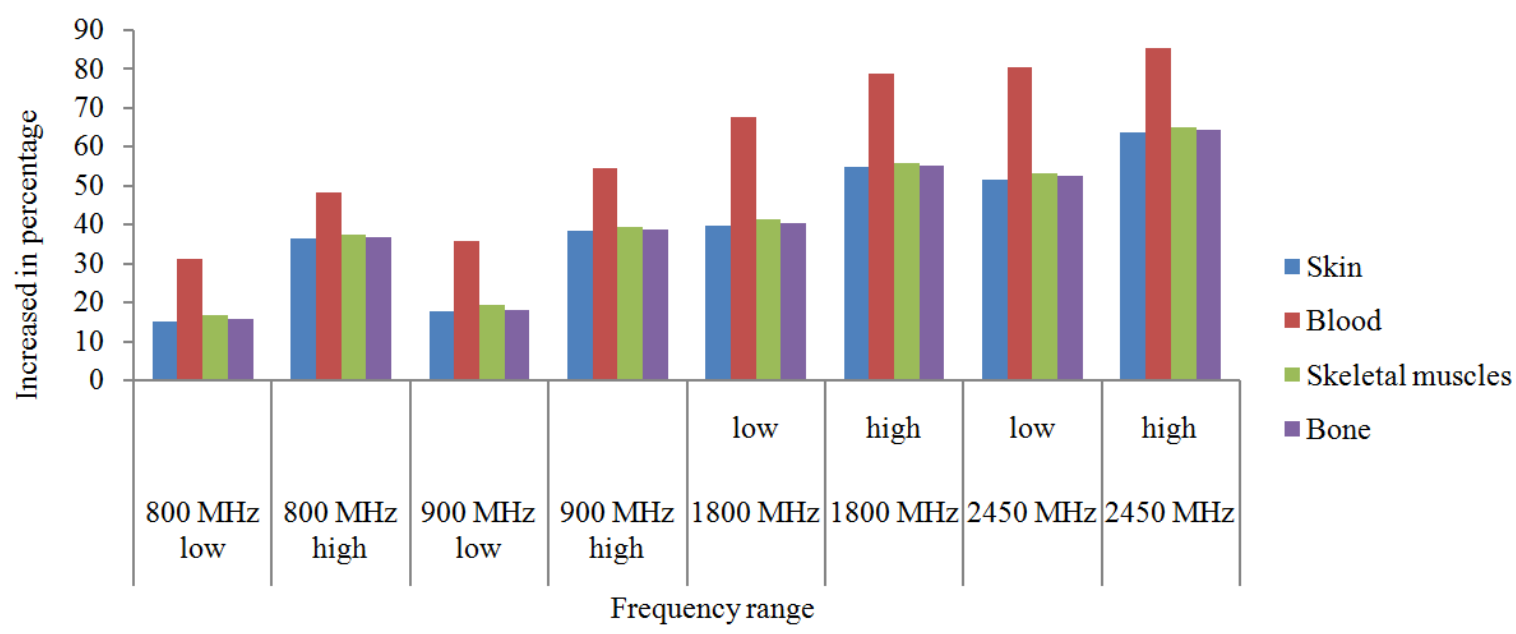

Fig. 1. Represents the increased percentage of skin, blood, muscles and bone tissues 


\section{Conclusion}

Four frequencies of mobile phone waves namely 800 , 900,1800 and $2450 \mathrm{MHz}$ for the calculation of change in temperature for skin, blood, muscles and bones at different depth and different distances are made.

When we move towards from $15 \mathrm{~cm}$ to $1 \mathrm{~cm}$ distance from mobile phone temperature of tissues increases. It is observed from the Table 1 to 16 at $800 \mathrm{MHz}$, temperature of skin increases from 15.16 to $36.36 \%$, temperature of blood increases from 31.10 to $48.32 \%$, for muscles from 16.68 to $37.51 \%$, for bone 15.63 to $36.72 \%$.
At frequency $900 \mathrm{MHz}$, temperature of skin increases from 17.71 to $38.29 \%$, temperature of blood increases from 35.66 to $54.40 \%$, for muscles from 19.26 to $39.47 \%$, for bone $18.21 \%$ to $38.68 \%$.

At frequency $1800 \mathrm{MHz}$, temperature of skin increases from 39.61 to $54.72 \%$, temperature of blood increases from 67.74 to $78.81 \%$, for muscles from 41.25 to $55.95 \%$, for bone 40.33 to $55.25 \%$.

At frequency $2450 \mathrm{MHz}$, temperature of skin increases from 51.66 to $63.78 \%$, temperature of blood increases from 80.34 to $85.27 \%$, for muscles from 53.36 to $65.07 \%$, for bone 52.54 to $64.45 \%$.

Table 1. Change in temperature (degree $\mathrm{C} / 15 \mathrm{~min}$ ) at different depth and $1 \mathrm{~cm}$ apart from the mobile phone at $800,900,1800$ and $2450 \mathrm{MHz}$

\begin{tabular}{|c|c|c|c|c|c|c|}
\hline \multirow[b]{2}{*}{ Frequency } & \multicolumn{6}{|c|}{ Change in temperature in $15 \mathrm{~min}, 1 \mathrm{~cm}$ distance from mobile phone at different depth } \\
\hline & Tissue name & $0.1 \mathrm{~mm}$ & $0.2 \mathrm{~mm}$ & $0.3 \mathrm{~mm}$ & $0.4 \mathrm{~mm}$ & $0.5 \mathrm{~mm}$ \\
\hline \multirow[t]{4}{*}{$800 \mathrm{MHz}$} & Skin & 1.868408 & 1.793178 & 1.720932 & 1.651645 & 1.58513 \\
\hline & Blood & 3.311267 & 3.028225 & 2.748348 & 2.503819 & 2.281137 \\
\hline & Muscles & 2.132611 & 2.037499 & 1.946575 & 1.859736 & 1.776734 \\
\hline & Bone & 0.213770 & 0.204871 & 0.196346 & 0.188181 & 0.180350 \\
\hline \multirow[t]{4}{*}{$900 \mathrm{MHz}$} & Skin & 1.936788 & 1.844612 & 1.756893 & 1.673333 & 1.593715 \\
\hline & Blood & 3.347286 & 2.997729 & 2.684838 & 2.404501 & 2.153485 \\
\hline & Muscles & 2.192846 & 2.078569 & 1.970315 & 1.867689 & 1.770394 \\
\hline & Bone & 0.230970 & 0.219644 & 0.208883 & 0.198648 & 0.188906 \\
\hline \multirow[t]{4}{*}{$1800 \mathrm{MHz}$} & Skin & 2.614534 & 2.304714 & 2.031655 & 1.790894 & 1.578688 \\
\hline & Blood & 3.742566 & 2.820563 & 2.125670 & 1.602042 & 1.207349 \\
\hline & Muscles & 2.880228 & 2.521550 & 2.207692 & 1.932750 & 1.692128 \\
\hline & Bone & 0.409922 & 0.360271 & 0.316641 & 0.278283 & 0.244580 \\
\hline \multirow[t]{4}{*}{$2450 \mathrm{MHz}$} & Skin & 3.191233 & 2.663020 & 2.219818 & 1.850446 & 1.542481 \\
\hline & Blood & 4.112398 & 2.740496 & 1.824355 & 1.214457 & 0.808453 \\
\hline & Muscles & 3.520391 & 2.911515 & 2.405322 & 1.987183 & 1.641702 \\
\hline & Bone & 0.554028 & 0.460126 & 0.381820 & 0.316836 & 0.262910 \\
\hline
\end{tabular}

Table 2. Change in temperature (degree $\mathrm{C} / 30 \mathrm{~min}$ ) at different depth and $1 \mathrm{~cm}$ apart from the mobile phone at $800,900,1800$ and $2450 \mathrm{MHz}$

\begin{tabular}{|c|c|c|c|c|c|c|}
\hline \multirow[b]{2}{*}{ Frequency } & \multicolumn{6}{|c|}{ Change in temperature in $30 \mathrm{~min}, 1 \mathrm{~cm}$ distance from mobile phone at different depth } \\
\hline & Tissue name & $0.1 \mathrm{~mm}$ & $0.2 \mathrm{~mm}$ & $0.3 \mathrm{~mm}$ & $0.4 \mathrm{~mm}$ & $0.5 \mathrm{~mm}$ \\
\hline \multirow[t]{4}{*}{$800 \mathrm{MHz}$} & Skin & 3.736815 & 3.586356 & 3.441864 & 3.303290 & 3.170260 \\
\hline & Blood & 6.622534 & 6.056450 & 5.496695 & 5.007638 & 4.562273 \\
\hline & Muscles & 4.265221 & 4.074998 & 3.893150 & 3.719472 & 3.553467 \\
\hline & Bone & 0.427539 & 0.409742 & 0.392691 & 0.376361 & 0.360701 \\
\hline \multirow[t]{4}{*}{$900 \mathrm{MHz}$} & Skin & 3.873576 & 3.689224 & 3.513787 & 3.346665 & 3.187431 \\
\hline & Blood & 6.694572 & 5.995458 & 5.369676 & 4.809001 & 4.306971 \\
\hline & Muscles & 4.385692 & 4.157138 & 3.940629 & 3.735377 & 3.540789 \\
\hline & Bone & 0.461940 & 0.439288 & 0.417765 & 0.397297 & 0.377812 \\
\hline \multirow[t]{4}{*}{$1800 \mathrm{MHz}$} & Skin & 5.229069 & 4.609428 & 4.063310 & 3.581788 & 3.157375 \\
\hline & Blood & 7.485132 & 5.641125 & 4.251339 & 3.204083 & 2.414699 \\
\hline & Muscles & 5.760456 & 5.043100 & 4.415384 & 3.865500 & 3.384255 \\
\hline & Bone & 0.819844 & 0.720543 & 0.633283 & 0.556566 & 0.489160 \\
\hline \multirow[t]{4}{*}{$2450 \mathrm{MHz}$} & Skin & 6.382466 & 5.326039 & 4.439636 & 3.700893 & 3.084961 \\
\hline & Blood & 8.224796 & 5.480991 & 3.648711 & 2.428913 & 1.616907 \\
\hline & Muscles & 7.040782 & 5.823030 & 4.810644 & 3.974366 & 3.283404 \\
\hline & Bone & 1.108056 & 0.920252 & 0.763639 & 0.633672 & 0.525820 \\
\hline
\end{tabular}


Table 3. Change in temperature (degree $\mathrm{C} / 45 \mathrm{~min}$ ) at different depth and $1 \mathrm{~cm}$ apart from the mobile phone at $800,900,1800$ and $2450 \mathrm{MHz}$

\begin{tabular}{|c|c|c|c|c|c|c|}
\hline \multirow[b]{2}{*}{ Frequency } & \multicolumn{6}{|c|}{ Change in temperature in $45 \mathrm{~min}, 1 \mathrm{~cm}$ distance from mobile phone at different depth } \\
\hline & Tissue name & $0.1 \mathrm{~mm}$ & $0.2 \mathrm{~mm}$ & $0.3 \mathrm{~mm}$ & $0.4 \mathrm{~mm}$ & $0.5 \mathrm{~mm}$ \\
\hline \multirow[t]{4}{*}{$800 \mathrm{MHz}$} & Skin & 5.605223 & 5.379534 & 5.162795 & 4.954935 & 4.755390 \\
\hline & Blood & 9.933801 & 9.084674 & 8.245043 & 7.511457 & 6.843410 \\
\hline & Muscles & 6.397832 & 6.112497 & 5.839725 & 5.579208 & 5.330201 \\
\hline & Bone & 0.641309 & 0.614614 & 0.589037 & 0.564542 & 0.541051 \\
\hline \multirow[t]{4}{*}{$900 \mathrm{MHz}$} & Skin & 5.810364 & 5.533836 & 5.270680 & 5.019998 & 4.781146 \\
\hline & Blood & 10.04186 & 8.993187 & 8.054514 & 7.213502 & 6.460456 \\
\hline & Muscles & 6.578538 & 6.235707 & 5.910944 & 5.603066 & 5.311183 \\
\hline & Bone & 0.692909 & 0.658932 & 0.626648 & 0.595945 & 0.566718 \\
\hline \multirow[t]{4}{*}{$1800 \mathrm{MHz}$} & Skin & 7.843603 & 6.914141 & 6.094964 & 5.372683 & 4.736063 \\
\hline & Blood & 11.22770 & 8.461688 & 6.377009 & 4.806125 & 3.622048 \\
\hline & Muscles & 8.640684 & 7.564651 & 6.623075 & 5.798249 & 5.076383 \\
\hline & Bone & 1.229766 & 1.080814 & 0.949924 & 0.834848 & 0.733740 \\
\hline \multirow[t]{4}{*}{$2450 \mathrm{MHz}$} & Skin & 9.573699 & 7.989059 & 6.659454 & 5.551339 & 4.627442 \\
\hline & Blood & 12.33719 & 8.221487 & 5.473066 & 3.643370 & 2.425360 \\
\hline & Muscles & 10.56117 & 8.734545 & 7.215966 & 5.961548 & 4.925106 \\
\hline & Bone & 1.662083 & 1.380379 & 1.145459 & 0.950508 & 0.788730 \\
\hline
\end{tabular}

Table 4. Change in temperature (degree $\mathrm{C} / 60 \mathrm{~min}$ ) at different depth and $1 \mathrm{~cm}$ apart from the mobile phone at 800, 900,1800 and $2450 \mathrm{MHz}$

\begin{tabular}{|c|c|c|c|c|c|c|}
\hline \multirow[b]{2}{*}{ Frequency } & \multicolumn{6}{|c|}{ Change in temperature in $60 \mathrm{~min}, 1 \mathrm{~cm}$ distance from mobile phone at different depth } \\
\hline & Tissue name & $0.1 \mathrm{~mm}$ & $0.2 \mathrm{~mm}$ & $0.3 \mathrm{~mm}$ & $0.4 \mathrm{~mm}$ & $0.5 \mathrm{~mm}$ \\
\hline \multirow[t]{4}{*}{$800 \mathrm{MHz}$} & Skin & 7.473631 & 7.172712 & 6.883727 & 6.606581 & 6.340519 \\
\hline & Blood & 13.24507 & 12.1129 & 10.99339 & 10.01528 & 9.124547 \\
\hline & Muscles & 8.530443 & 8.149996 & 7.786299 & 7.438943 & 7.106934 \\
\hline & Bone & 0.855078 & 0.819485 & 0.785382 & 0.752723 & 0.721402 \\
\hline \multirow[t]{4}{*}{$900 \mathrm{MHz}$} & Skin & 7.747152 & 7.378449 & 7.027573 & 6.693331 & 6.374861 \\
\hline & Blood & 13.38914 & 11.99092 & 10.73935 & 9.618003 & 8.613942 \\
\hline & Muscles & 8.771384 & 8.314277 & 7.881258 & 7.470754 & 7.081578 \\
\hline & Bone & 0.923879 & 0.878575 & 0.835531 & 0.794594 & 0.755624 \\
\hline \multirow[t]{4}{*}{$1800 \mathrm{MHz}$} & Skin & 10.45814 & 9.218855 & 8.126619 & 7.163577 & 6.314750 \\
\hline & Blood & 14.97026 & 11.28225 & 8.502679 & 6.408167 & 4.829397 \\
\hline & Muscles & 11.52091 & 10.08620 & 8.830767 & 7.730999 & 6.768511 \\
\hline & Bone & 1.639688 & 1.441085 & 1.266565 & 1.113131 & 0.978319 \\
\hline \multirow[t]{4}{*}{$2450 \mathrm{MHz}$} & Skin & 12.76493 & 10.65208 & 8.879272 & 7.401786 & 6.169923 \\
\hline & Blood & 16.44959 & 10.96198 & 7.297421 & 4.857827 & 3.233813 \\
\hline & Muscles & 14.08156 & 11.64606 & 9.621288 & 7.948731 & 6.566809 \\
\hline & Bone & 2.216111 & 1.840505 & 1.527278 & 1.267344 & 1.051640 \\
\hline
\end{tabular}

Table 5. Change in temperature (degree $\mathrm{C} / 15 \mathrm{~min}$ ) at different depth and $5 \mathrm{~cm}$ apart from the mobile phone at $800,900,1800$ and $2450 \mathrm{MHz}$

Change in temperature in $15 \mathrm{~min}, 5 \mathrm{~cm}$ distance from mobile phone at different depth

\begin{tabular}{lllllll} 
Frequency & Tissue name & $0.1 \mathrm{~mm}$ & $0.2 \mathrm{~mm}$ & $0.3 \mathrm{~mm}$ & $0.4 \mathrm{~mm}$ & $0.5 \mathrm{~mm}$ \\
\hline $800 \mathrm{MHz}$ & Skin & 0.074732 & 0.071724 & 0.068832 & 0.066064 & 0.063405 \\
& Blood & 0.132433 & 0.120664 & 0.109915 & 0.100142 & 0.091243 \\
& Muscles & 0.085300 & 0.081491 & 0.077854 & 0.074382 & 0.071069 \\
& Bone & 0.008550 & 0.008194 & 0.007853 & 0.007527 & 0.007214 \\
$900 \mathrm{MHz}$ & Skin & 0.077472 & 0.073782 & 0.070272 & 0.066484 & 0.063745 \\
& Blood & 0.140567 & 0.119894 & 0.107384 & 0.095530 & 0.086133 \\
& Muscles & 0.087714 & 0.083133 & 0.078808 & 0.074204 & 0.070807 \\
& Bone & 0.009238 & 0.008785 & 0.008354 & 0.007893 & 0.007555 \\
$1800 \mathrm{MHz}$ & Skin & 0.104573 & 0.092183 & 0.081263 & 0.071636 & 0.063144 \\
& Blood & 0.149677 & 0.112803 & 0.085020 & 0.064082 & 0.048292 \\
& Muscles & 0.115193 & 0.100856 & 0.088296 & 0.077306 & 0.067675 \\
& Bone & 0.016395 & 0.014409 & 0.012665 & 0.011131 & 0.009782 \\
& Skin & 0.127782 & 0.106514 & 0.088793 & 0.074010 & 0.061696 \\
& Blood & 0.164665 & 0.109605 & 0.072972 & 0.048566 & 0.032336 \\
& Muscles & 0.140954 & 0.116450 & 0.096206 & 0.079481 & 0.065660 \\
& Bone & 0.022183 & 0.018404 & 0.015271 & 0.012672 & 0.010516 \\
\hline
\end{tabular}


Table 6. Change in temperature (degree $\mathrm{C} / 30 \mathrm{~min}$ ) at different depth and $5 \mathrm{~cm}$ apart from the mobile phone at $800,900,1800$ and $2450 \mathrm{MHz}$

\begin{tabular}{|c|c|c|c|c|c|c|}
\hline \multirow[b]{2}{*}{ Frequency } & \multicolumn{6}{|c|}{ Change in temperature in $30 \mathrm{~min}, 5 \mathrm{~cm}$ distance from mobile phone at different depth } \\
\hline & Tissue name & $0.1 \mathrm{~mm}$ & $0.2 \mathrm{~mm}$ & $0.3 \mathrm{~mm}$ & $0.4 \mathrm{~mm}$ & $0.5 \mathrm{~mm}$ \\
\hline \multirow[t]{4}{*}{$800 \mathrm{MHz}$} & Skin & 0.149464 & 0.143449 & 0.137664 & 0.132129 & 0.126810 \\
\hline & Blood & 0.264866 & 0.241328 & 0.219831 & 0.200283 & 0.182487 \\
\hline & Muscles & 0.170601 & 0.162981 & 0.155708 & 0.148764 & 0.142139 \\
\hline & Bone & 0.017100 & 0.016388 & 0.015706 & 0.015054 & 0.014427 \\
\hline \multirow[t]{4}{*}{$900 \mathrm{MHz}$} & Skin & 0.154943 & 0.147563 & 0.140543 & 0.132968 & 0.127489 \\
\hline & Blood & 0.281134 & 0.239789 & 0.214769 & 0.191061 & 0.172266 \\
\hline & Muscles & 0.175428 & 0.166266 & 0.157616 & 0.148408 & 0.141614 \\
\hline & Bone & 0.018477 & 0.017571 & 0.016709 & 0.015785 & 0.015111 \\
\hline \multirow[t]{4}{*}{$1800 \mathrm{MHz}$} & Skin & 0.209147 & 0.184366 & 0.162525 & 0.143272 & 0.126289 \\
\hline & Blood & 0.299355 & 0.225607 & 0.170039 & 0.128163 & 0.096584 \\
\hline & Muscles & 0.230387 & 0.201711 & 0.176592 & 0.154613 & 0.135350 \\
\hline & Bone & 0.032791 & 0.028819 & 0.025330 & 0.022261 & 0.019565 \\
\hline \multirow[t]{4}{*}{$2450 \mathrm{MHz}$} & Skin & 0.255564 & 0.213028 & 0.177585 & 0.148020 & 0.123391 \\
\hline & Blood & 0.329331 & 0.219210 & 0.145943 & 0.097132 & 0.064673 \\
\hline & Muscles & 0.281909 & 0.232901 & 0.192412 & 0.158962 & 0.131321 \\
\hline & Bone & 0.044366 & 0.036808 & 0.030543 & 0.025344 & 0.021032 \\
\hline
\end{tabular}

Table 7. Change in temperature (degree $\mathrm{C} / 45 \mathrm{~min}$ ) at different depth and $5 \mathrm{~cm}$ apart from the mobile phone at $800,900,1800$ and $2450 \mathrm{MHz}$

\begin{tabular}{|c|c|c|c|c|c|c|}
\hline \multirow[b]{2}{*}{ Frequency } & \multicolumn{6}{|c|}{ Change in temperature in $45 \mathrm{~min}, 5 \mathrm{~cm}$ distance from mobile phone at different depth } \\
\hline & Tissue name & $0.1 \mathrm{~mm}$ & $0.2 \mathrm{~mm}$ & $0.3 \mathrm{~mm}$ & $0.4 \mathrm{~mm}$ & $0.5 \mathrm{~mm}$ \\
\hline \multirow[t]{4}{*}{$800 \mathrm{MHz}$} & Skin & 0.224196 & 0.215173 & 0.206496 & 0.198193 & 0.190216 \\
\hline & Blood & 0.397299 & 0.361992 & 0.329746 & 0.300425 & 0.273730 \\
\hline & Muscles & 0.255901 & 0.244472 & 0.233562 & 0.223146 & 0.213208 \\
\hline & Bone & 0.025650 & 0.024582 & 0.023560 & 0.022581 & 0.021641 \\
\hline \multirow[t]{4}{*}{$900 \mathrm{MHz}$} & Skin & 0.232415 & 0.221345 & 0.210815 & 0.199451 & 0.191234 \\
\hline & Blood & 0.421701 & 0.359683 & 0.322153 & 0.286591 & 0.258400 \\
\hline & Muscles & 0.263141 & 0.249399 & 0.236424 & 0.222612 & 0.212421 \\
\hline & Bone & 0.027715 & 0.026356 & 0.025063 & 0.023678 & 0.022666 \\
\hline \multirow[t]{4}{*}{$1800 \mathrm{MHz}$} & Skin & 0.313720 & 0.276548 & 0.243788 & 0.214907 & 0.189433 \\
\hline & Blood & 0.449032 & 0.338410 & 0.255059 & 0.192245 & 0.144877 \\
\hline & Muscles & 0.345580 & 0.302567 & 0.264887 & 0.231919 & 0.203024 \\
\hline & Bone & 0.049186 & 0.043228 & 0.037995 & 0.033392 & 0.029347 \\
\hline \multirow[t]{4}{*}{$2450 \mathrm{MHz}$} & Skin & 0.383347 & 0.319541 & 0.266378 & 0.222030 & 0.185087 \\
\hline & Blood & 0.493996 & 0.328814 & 0.218915 & 0.145699 & 0.097009 \\
\hline & Muscles & 0.422863 & 0.349351 & 0.288618 & 0.238443 & 0.196981 \\
\hline & Bone & 0.066549 & 0.055211 & 0.045814 & 0.038015 & 0.031547 \\
\hline \multicolumn{7}{|c|}{$\begin{array}{l}\text { Table 8. Change in temperature (degree } \mathrm{C} / 60 \mathrm{~min} \text { ) at different depth and } 5 \mathrm{~cm} \text { apart from the mobile phone at } 800,900,1800 \text { and } \\
2450 \mathrm{MHz}\end{array}$} \\
\hline & \multicolumn{6}{|c|}{ Change in temperature in $60 \mathrm{mint}, 5 \mathrm{~cm}$ distance from mobile phone at different depth } \\
\hline Frequency & Tissue name & $0.1 \mathrm{~mm}$ & $0.2 \mathrm{~mm}$ & $0.3 \mathrm{~mm}$ & $0.4 \mathrm{~mm}$ & $0.5 \mathrm{~mm}$ \\
\hline \multirow[t]{4}{*}{$800 \mathrm{MHz}$} & Skin & 0.298928 & 0.286898 & 0.275328 & 0.264258 & 0.253621 \\
\hline & Blood & 0.529732 & 0.482656 & 0.439662 & 0.400567 & 0.364974 \\
\hline & Muscles & 0.341202 & 0.325962 & 0.311415 & 0.297528 & 0.284277 \\
\hline & Bone & 0.034201 & 0.032776 & 0.031413 & 0.030108 & 0.028855 \\
\hline \multirow[t]{4}{*}{$900 \mathrm{MHz}$} & Skin & 0.309886 & 0.295127 & 0.281086 & 0.265935 & 0.254979 \\
\hline & Blood & 0.562268 & 0.479578 & 0.429537 & 0.382122 & 0.344533 \\
\hline & Muscles & 0.350855 & 0.332533 & 0.315232 & 0.296816 & 0.283228 \\
\hline & Bone & 0.036953 & 0.035141 & 0.033417 & 0.031570 & 0.030222 \\
\hline \multirow[t]{4}{*}{$1800 \mathrm{MHz}$} & Skin & 0.418293 & 0.368731 & 0.325050 & 0.286543 & 0.252577 \\
\hline & Blood & 0.598710 & 0.451213 & 0.340079 & 0.256327 & 0.193169 \\
\hline & Muscles & 0.460774 & 0.403423 & 0.353183 & 0.309225 & 0.270699 \\
\hline & Bone & 0.065581 & 0.057637 & 0.050660 & 0.044522 & 0.039130 \\
\hline \multirow[t]{4}{*}{$2450 \mathrm{MHz}$} & Skin & 0.511129 & 0.426055 & 0.355171 & 0.296040 & 0.246783 \\
\hline & Blood & 0.658662 & 0.438419 & 0.291887 & 0.194265 & 0.129346 \\
\hline & Muscles & 0.563818 & 0.465801 & 0.384823 & 0.317924 & 0.262641 \\
\hline & Bone & 0.088732 & 0.073615 & 0.061085 & 0.050687 & 0.042063 \\
\hline
\end{tabular}


Table 9. Change in temperature (degree $\mathrm{C} / 15 \mathrm{~min}$ ) at different depth and $10 \mathrm{~cm}$ apart from the mobile phone at $800,900,1800$ and $2450 \mathrm{MHz}$

\begin{tabular}{|c|c|c|c|c|c|c|}
\hline \multirow[b]{2}{*}{ Frequency } & \multicolumn{6}{|c|}{ Change in temperature in $15 \mathrm{~min}, 10 \mathrm{~cm}$ distance from mobile phone at different depth } \\
\hline & Tissue name & $0.1 \mathrm{~mm}$ & $0.2 \mathrm{~mm}$ & $0.3 \mathrm{~mm}$ & $0.4 \mathrm{~mm}$ & $0.5 \mathrm{~mm}$ \\
\hline \multirow[t]{4}{*}{$800 \mathrm{MHz}$} & Skin & 0.018683 & 0.017931 & 0.017208 & 0.016516 & 0.015851 \\
\hline & Blood & 0.033108 & 0.030171 & 0.027480 & 0.025033 & 0.022810 \\
\hline & Muscles & 0.021323 & 0.020373 & 0.019463 & 0.018594 & 0.017766 \\
\hline & Bone & 0.002137 & 0.002049 & 0.001963 & 0.001882 & 0.001803 \\
\hline \multirow[t]{4}{*}{$900 \mathrm{MHz}$} & Skin & 0.019366 & 0.018445 & 0.017568 & 0.016732 & 0.015936 \\
\hline & Blood & 0.035142 & 0.029971 & 0.026846 & 0.024041 & 0.021531 \\
\hline & Muscles & 0.021924 & 0.020783 & 0.019700 & 0.018672 & 0.017702 \\
\hline & Bone & 0.002309 & 0.002196 & 0.002088 & 0.001986 & 0.001889 \\
\hline \multirow[t]{4}{*}{$1800 \mathrm{MHz}$} & Skin & 0.026141 & 0.023043 & 0.020316 & 0.017907 & 0.015784 \\
\hline & Blood & 0.037415 & 0.028197 & 0.021255 & 0.016015 & 0.012069 \\
\hline & Muscles & 0.028793 & 0.025211 & 0.022074 & 0.019324 & 0.016917 \\
\hline & Bone & 0.004098 & 0.003602 & 0.003166 & 0.002782 & 0.002445 \\
\hline \multirow[t]{4}{*}{$2450 \mathrm{MHz}$} & Skin & 0.031942 & 0.026628 & 0.022196 & 0.018500 & 0.015424 \\
\hline & Blood & 0.041162 & 0.027401 & 0.018237 & 0.012142 & 0.008082 \\
\hline & Muscles & 0.034952 & 0.029109 & 0.024046 & 0.019868 & 0.016415 \\
\hline & Bone & 0.005545 & 0.004611 & 0.003817 & 0.003168 & 0.002629 \\
\hline
\end{tabular}

Table 10. Change in temperature (degree $\mathrm{C} / 30 \mathrm{~min}$ ) at different depth and $10 \mathrm{~cm}$ apart from the mobile phone at $800,900,1800$ and $2450 \mathrm{MHz}$

\begin{tabular}{|c|c|c|c|c|c|c|}
\hline \multirow[b]{2}{*}{ Frequency } & \multicolumn{6}{|c|}{ Change in temperature in $30 \mathrm{~min}, 10 \mathrm{~cm}$ distance from mobile phone at different depth } \\
\hline & Tissue name & $0.1 \mathrm{~mm}$ & $0.2 \mathrm{~mm}$ & $0.3 \mathrm{~mm}$ & $0.4 \mathrm{~mm}$ & $0.5 \mathrm{~mm}$ \\
\hline \multirow[t]{4}{*}{$800 \mathrm{MHz}$} & Skin & 0.037366 & 0.035862 & 0.034416 & 0.033032 & 0.031703 \\
\hline & Blood & 0.066216 & 0.060343 & 0.054960 & 0.050065 & 0.045621 \\
\hline & Muscles & 0.042645 & 0.040745 & 0.038927 & 0.037187 & 0.035531 \\
\hline & Bone & 0.004275 & 0.004097 & 0.003926 & 0.003764 & 0.003606 \\
\hline \multirow[t]{4}{*}{$900 \mathrm{MHz}$} & Skin & 0.038732 & 0.036891 & 0.035136 & 0.033464 & 0.031872 \\
\hline & Blood & 0.070284 & 0.059941 & 0.053692 & 0.048082 & 0.043061 \\
\hline & Muscles & 0.043849 & 0.041567 & 0.039400 & 0.037345 & 0.035403 \\
\hline & Bone & 0.004618 & 0.004393 & 0.004177 & 0.003972 & 0.003778 \\
\hline \multirow{4}{*}{$1800 \mathrm{MHz}$} & Skin & 0.052282 & 0.046087 & 0.040631 & 0.035814 & 0.031568 \\
\hline & Blood & 0.074831 & 0.056395 & 0.042510 & 0.032031 & 0.024137 \\
\hline & Muscles & 0.057585 & 0.050423 & 0.044148 & 0.038649 & 0.033833 \\
\hline & Bone & 0.008196 & 0.007204 & 0.006332 & 0.005565 & 0.004890 \\
\hline \multirow[t]{4}{*}{$2450 \mathrm{MHz}$} & Skin & 0.063885 & 0.053257 & 0.044391 & 0.037000 & 0.030848 \\
\hline & Blood & 0.082324 & 0.054802 & 0.036474 & 0.024283 & 0.016164 \\
\hline & Muscles & 0.069905 & 0.058219 & 0.048091 & 0.039735 & 0.032830 \\
\hline & Bone & 0.011090 & 0.009221 & 0.007635 & 0.006335 & 0.005257 \\
\hline
\end{tabular}

Table 11. Change in temperature (degree $\mathrm{C} / 45 \mathrm{~min}$ ) at different depth and $10 \mathrm{~cm}$ apart from the mobile phone at $800,900,1800$ and $2450 \mathrm{MHz}$

Change in temperature in $45 \mathrm{~min}, 10 \mathrm{~cm}$ distance from mobile phone at different depth

\begin{tabular}{lllllll} 
Frequency & Tissue name & $0.1 \mathrm{~mm}$ & $0.2 \mathrm{~mm}$ & $0.3 \mathrm{~mm}$ & $0.4 \mathrm{~mm}$ & $0.5 \mathrm{~mm}$ \\
\hline $800 \mathrm{MHz}$ & Skin & 0.056049 & 0.053793 & 0.051624 & 0.049548 & 0.047554 \\
& Blood & 0.099325 & 0.090514 & 0.082440 & 0.075098 & 0.068431 \\
& Muscles & 0.063968 & 0.061118 & 0.058390 & 0.055781 & 0.053297 \\
& Bone & 0.006412 & 0.006146 & 0.005889 & 0.005645 & 0.005410 \\
$900 \mathrm{MHz}$ & Skin & 0.058098 & 0.055336 & 0.052704 & 0.050196 & 0.047808 \\
& Blood & 0.105425 & 0.089912 & 0.080538 & 0.072124 & 0.064592 \\
& Muscles & 0.065773 & 0.062350 & 0.059100 & 0.056017 & 0.053105 \\
& Bone & 0.006927 & 0.006589 & 0.006265 & 0.005958 & 0.005667 \\
$1800 \mathrm{MHz}$ & Skin & 0.078422 & 0.069130 & 0.060947 & 0.053721 & 0.047352 \\
& Blood & 0.112246 & 0.084592 & 0.063765 & 0.048046 & 0.036206 \\
& Muscles & 0.086378 & 0.075634 & 0.066222 & 0.057973 & 0.050750 \\
& Bone & 0.012294 & 0.010806 & 0.009498 & 0.008347 & 0.007335 \\
& Skin & 0.095827 & 0.079885 & 0.066587 & 0.055500 & 0.046272 \\
& Blood & 0.123485 & 0.082204 & 0.054710 & 0.036425 & 0.024246 \\
& Muscles & 0.104857 & 0.087328 & 0.072137 & 0.059603 & 0.049245 \\
& Bone & 0.016636 & 0.013832 & 0.011452 & 0.009503 & 0.007886 \\
\hline
\end{tabular}


Table 12. Change in temperature (degree $\mathrm{C} / 60 \mathrm{~min}$ ) at different depth and $10 \mathrm{~cm}$ apart from the mobile phone at $800,900,1800$ and $2450 \mathrm{MHz}$

\begin{tabular}{|c|c|c|c|c|c|c|}
\hline \multirow[b]{2}{*}{ Frequency } & \multicolumn{6}{|c|}{ Change in temperature in $60 \mathrm{~min}, 10 \mathrm{~cm}$ distance from mobile phone at different depth } \\
\hline & Tissue name & $0.1 \mathrm{~mm}$ & $0.2 \mathrm{~mm}$ & $0.3 \mathrm{~mm}$ & $0.4 \mathrm{~mm}$ & $0.5 \mathrm{~mm}$ \\
\hline \multirow[t]{4}{*}{$800 \mathrm{MHz}$} & Skin & 0.074732 & 0.071724 & 0.068832 & 0.066064 & 0.063405 \\
\hline & Blood & 0.132433 & 0.120686 & 0.109920 & 0.100131 & 0.091241 \\
\hline & Muscles & 0.085290 & 0.081491 & 0.077854 & 0.074375 & 0.071062 \\
\hline & Bone & 0.008549 & 0.008194 & 0.007852 & 0.007527 & 0.007213 \\
\hline \multirow[t]{4}{*}{$900 \mathrm{MHz}$} & Skin & 0.077464 & 0.073782 & 0.070272 & 0.066928 & 0.063745 \\
\hline & Blood & 0.140567 & 0.119882 & 0.107384 & 0.096165 & 0.086123 \\
\hline & Muscles & 0.087697 & 0.083133 & 0.078800 & 0.074689 & 0.070807 \\
\hline & Bone & 0.009237 & 0.008785 & 0.008353 & 0.007944 & 0.007555 \\
\hline \multirow[t]{4}{*}{$1800 \mathrm{MHz}$} & Skin & 0.104563 & 0.092173 & 0.081263 & 0.071627 & 0.063136 \\
\hline & Blood & 0.149662 & 0.112790 & 0.085020 & 0.064061 & 0.048274 \\
\hline & Muscles & 0.115171 & 0.100845 & 0.088296 & 0.077297 & 0.067666 \\
\hline & Bone & 0.016392 & 0.014408 & 0.012664 & 0.011129 & 0.009780 \\
\hline \multirow[t]{4}{*}{$2450 \mathrm{MHz}$} & Skin & 0.127769 & 0.106514 & 0.088782 & 0.074000 & 0.061696 \\
\hline & Blood & 0.164647 & 0.109605 & 0.072947 & 0.048566 & 0.032328 \\
\hline & Muscles & 0.139809 & 0.116437 & 0.096182 & 0.079470 & 0.065660 \\
\hline & Bone & 0.022181 & 0.018442 & 0.015269 & 0.012670 & 0.010514 \\
\hline
\end{tabular}

Table 13. Change in temperature (degree $\mathrm{C} / 15 \mathrm{~min}$ ) at different depth and $15 \mathrm{~cm}$ apart from the mobile phone at $800,900,1800$ and $2450 \mathrm{MHz}$

Change in temperature in $15 \mathrm{~min}, 15 \mathrm{~cm}$ distance from mobile phone at different depth

\begin{tabular}{lllllll}
\multirow{2}{*}{ Frequency } & Tissue name & $0.1 \mathrm{~mm}$ & $0.2 \mathrm{~mm}$ & $0.3 \mathrm{~mm}$ & $0.4 \mathrm{~mm}$ & $0.5 \mathrm{~mm}$ \\
\hline $800 \mathrm{MHz}$ & Skin & 0.008302 & 0.007969 & 0.007647 & 0.007340 & 0.007044 \\
& Blood & 0.014712 & 0.014518 & 0.012209 & 0.011126 & 0.010137 \\
& Muscles & 0.009474 & 0.009054 & 0.008648 & 0.008264 & 0.007893 \\
& Bone & 0.000950 & 0.000910 & 0.000872 & 0.000836 & 0.000801 \\
$900 \mathrm{MHz}$ & Skin & 0.008606 & 0.008196 & 0.007806 & 0.007436 & 0.007081 \\
& Blood & 0.015614 & 0.013318 & 0.011929 & 0.010683 & 0.009567 \\
& Muscles & 0.009744 & 0.009233 & 0.008753 & 0.008299 & 0.007864 \\
& Bone & 0.001026 & 0.000976 & 0.000928 & 0.000882 & 0.000839 \\
$1800 \mathrm{MHz}$ & Skin & 0.011619 & 0.010241 & 0.009028 & 0.007959 & 0.007014 \\
& Blood & 0.016629 & 0.012534 & 0.009443 & 0.007118 & 0.005363 \\
& Muscles & 0.012798 & 0.011205 & 0.009810 & 0.008587 & 0.007517 \\
& Bone & 0.001821 & 0.001601 & 0.001407 & 0.001237 & 0.001087 \\
& Skin & 0.010987 & 0.009157 & 0.007634 & 0.006363 & 0.005305 \\
& Blood & 0.018292 & 0.012177 & 0.008103 & 0.005395 & 0.003592 \\
& Muscles & 0.015660 & 0.012933 & 0.008830 & 0.008830 & 0.007293 \\
& Bone & 0.008302 & 0.007969 & 0.007647 & 0.007340 & 0.007044 \\
\hline
\end{tabular}

Table 14. Change in temperature (degree $\mathrm{C} / 30 \mathrm{~min}$ ) at different depth and $15 \mathrm{~cm}$ apart from the mobile phone at $800,900,1800$ and $2450 \mathrm{MHz}$

Change in temperature in $30 \mathrm{~min}, 15 \mathrm{~cm}$ distance from mobile phone at different depth

\begin{tabular}{lllllll} 
Frequency & Tissue name & $0.1 \mathrm{~mm}$ & $0.2 \mathrm{~mm}$ & $0.3 \mathrm{~mm}$ & $0.4 \mathrm{~mm}$ & $0.5 \mathrm{~mm}$ \\
\hline $800 \mathrm{MHz}$ & Skin & 0.016604 & 0.015937 & 0.015293 & 0.014680 & 0.014087 \\
& Blood & 0.029424 & 0.029036 & 0.024418 & 0.022251 & 0.020274 \\
& Muscles & 0.018948 & 0.018107 & 0.017296 & 0.016528 & 0.015787 \\
& Bone & 0.001900 & 0.001821 & 0.001744 & 0.001672 & 0.001603 \\
$900 \mathrm{MHz}$ & Skin & 0.017213 & 0.016391 & 0.015613 & 0.014871 & 0.014161 \\
& Blood & 0.031228 & 0.026635 & 0.023858 & 0.021365 & 0.019134 \\
& Muscles & 0.019488 & 0.018465 & 0.017506 & 0.016598 & 0.015728 \\
& Bone & 0.002052 & 0.001952 & 0.001856 & 0.001765 & 0.001678 \\
$1800 \mathrm{MHz}$ & Skin & 0.023239 & 0.020483 & 0.018056 & 0.015917 & 0.014029 \\
& Blood & 0.033258 & 0.025067 & 0.018885 & 0.014236 & 0.010726 \\
& Muscles & 0.025596 & 0.022410 & 0.019619 & 0.017173 & 0.015033 \\
& Bone & 0.003643 & 0.003201 & 0.002814 & 0.002473 & 0.002173 \\
& Skin & 0.021974 & 0.018314 & 0.015267 & 0.012727 & 0.010609 \\
& Blood & 0.036584 & 0.024353 & 0.016205 & 0.010790 & 0.007184 \\
& Muscles & 0.031320 & 0.025866 & 0.017660 & 0.017660 & 0.014587 \\
& Bone & 0.004929 & 0.004088 & 0.003393 & 0.002816 & 0.002336 \\
\hline
\end{tabular}


Table 15. Change in temperature (degree $\mathrm{C} / 45 \mathrm{~min}$ ) at different depth and $15 \mathrm{~cm}$ apart from the mobile phone at $800,900,1800$ and $2450 \mathrm{MHz}$

\begin{tabular}{|c|c|c|c|c|c|c|}
\hline \multirow[b]{2}{*}{ Frequency } & \multicolumn{6}{|c|}{ Change in temperature in $45 \mathrm{~min}, 15 \mathrm{~cm}$ distance from mobile phone at different depth } \\
\hline & Tissue name & $0.1 \mathrm{~mm}$ & $0.2 \mathrm{~mm}$ & $0.3 \mathrm{~mm}$ & $0.4 \mathrm{~mm}$ & $0.5 \mathrm{~mm}$ \\
\hline \multirow[t]{4}{*}{$800 \mathrm{MHz}$} & Skin & 0.024906 & 0.023906 & 0.022940 & 0.022019 & 0.021131 \\
\hline & Blood & 0.044136 & 0.043553 & 0.036627 & 0.033377 & 0.030411 \\
\hline & Muscles & 0.028422 & 0.027161 & 0.025944 & 0.024792 & 0.023680 \\
\hline & Bone & 0.002850 & 0.002731 & 0.002617 & 0.002509 & 0.002404 \\
\hline \multirow[t]{4}{*}{$900 \mathrm{MHz}$} & Skin & 0.025819 & 0.024587 & 0.023419 & 0.022307 & 0.021242 \\
\hline & Blood & 0.046842 & 0.039953 & 0.035787 & 0.032048 & 0.028701 \\
\hline & Muscles & 0.029232 & 0.027698 & 0.026259 & 0.024896 & 0.023592 \\
\hline & Bone & 0.003079 & 0.002927 & 0.002784 & 0.002647 & 0.002517 \\
\hline \multirow[t]{4}{*}{$1800 \mathrm{MHz}$} & Skin & 0.034858 & 0.030724 & 0.027085 & 0.023876 & 0.021043 \\
\hline & Blood & 0.049887 & 0.037601 & 0.028328 & 0.021354 & 0.016088 \\
\hline & Muscles & 0.038394 & 0.033615 & 0.029429 & 0.025760 & 0.022550 \\
\hline & Bone & 0.005464 & 0.004802 & 0.004221 & 0.003710 & 0.003260 \\
\hline \multirow[t]{4}{*}{$2450 \mathrm{MHz}$} & Skin & 0.032961 & 0.027472 & 0.022901 & 0.019090 & 0.015914 \\
\hline & Blood & 0.054876 & 0.036530 & 0.024308 & 0.016185 & 0.010776 \\
\hline & Muscles & 0.046980 & 0.038800 & 0.026490 & 0.026490 & 0.021880 \\
\hline & Bone & 0.024906 & 0.023906 & 0.022940 & 0.022019 & 0.021131 \\
\hline
\end{tabular}

Table 16. Change in temperature (degree $\mathrm{C} / 60 \mathrm{~min}$ ) at different depth and $15 \mathrm{~cm}$ apart from the mobile phone at $800,900,1800$ and $2450 \mathrm{MHz}$

\begin{tabular}{|c|c|c|c|c|c|c|}
\hline \multirow[b]{2}{*}{ Frequency } & \multicolumn{6}{|c|}{ Change in temperature in $60 \mathrm{~min}, 15 \mathrm{~cm}$ distance from mobile phone at different depth } \\
\hline & Tissue name & $0.1 \mathrm{~mm}$ & $0.2 \mathrm{~mm}$ & $0.3 \mathrm{~mm}$ & $0.4 \mathrm{~mm}$ & $0.5 \mathrm{~mm}$ \\
\hline \multirow[t]{4}{*}{$800 \mathrm{MHz}$} & Skin & 0.033208 & 0.031874 & 0.030586 & 0.022019 & 0.021131 \\
\hline & Blood & 0.058848 & 0.058071 & 0.048836 & 0.033377 & 0.030411 \\
\hline & Muscles & 0.037896 & 0.036215 & 0.034592 & 0.024792 & 0.023680 \\
\hline & Bone & 0.003799 & 0.003641 & 0.003489 & 0.002509 & 0.002404 \\
\hline \multirow[t]{4}{*}{$900 \mathrm{MHz}$} & Skin & 0.034425 & 0.032782 & 0.031226 & 0.022307 & 0.021242 \\
\hline & Blood & 0.062457 & 0.053270 & 0.047716 & 0.032048 & 0.028701 \\
\hline & Muscles & 0.038977 & 0.036930 & 0.035012 & 0.024896 & 0.023592 \\
\hline & Bone & 0.004105 & 0.003903 & 0.003712 & 0.002647 & 0.002517 \\
\hline \multirow[t]{4}{*}{$1800 \mathrm{MHz}$} & Skin & 0.046477 & 0.040966 & 0.036113 & 0.023876 & 0.021043 \\
\hline & Blood & 0.066516 & 0.050135 & 0.037771 & 0.021354 & 0.016088 \\
\hline & Muscles & 0.051192 & 0.044820 & 0.039238 & 0.025760 & 0.022550 \\
\hline & Bone & 0.007285 & 0.006402 & 0.005628 & 0.003710 & 0.003260 \\
\hline \multirow[t]{4}{*}{$2450 \mathrm{MHz}$} & Skin & 0.043948 & 0.036629 & 0.030534 & 0.019090 & 0.015914 \\
\hline & Blood & 0.073168 & 0.048707 & 0.032410 & 0.016185 & 0.010776 \\
\hline & Muscles & 0.062640 & 0.051733 & 0.035320 & 0.026490 & 0.021880 \\
\hline & Bone & 0.009857 & 0.008177 & 0.006786 & 0.004223 & 0.003504 \\
\hline
\end{tabular}

Table 17. Represents the increased percentage of skin blood, muscles and bone tissue at frequencies of $800,900,1800 \mathrm{and} 2450 \mathrm{MHz}$

\begin{tabular}{|c|c|c|c|c|}
\hline Name of the tissues & $\begin{array}{l}\text { Increased } \% \text { at frequency } \\
800 \mathrm{MHz} \text { (handset) }\end{array}$ & $\begin{array}{l}\text { Increased \% at frequency } \\
900 \mathrm{MHz} \text { (handset) }\end{array}$ & $\begin{array}{l}\text { Increased \% at frequency } \\
1800 \mathrm{MHz} \text { (handset) }\end{array}$ & $\begin{array}{l}\text { Increased \% at frequency } \\
2450 \mathrm{MHz} \text { (handset) }\end{array}$ \\
\hline Skin & 15.16 to 36.36 & 17.71 to 38.29 & 39.61 to 54.72 & 51.66 to 63.78 \\
\hline Blood & 31.10 to 48.32 & 35.66 to 54.40 & 67.74 to 78.81 & 80.34 to 85.27 \\
\hline Skeletal muscles & 16.68 to 37.51 & 19.26 to 39.47 & 41.25 to 55.95 & 53.36 to 65.07 \\
\hline Bone & 15.63 to 36.72 & 18.21 to 38.68 & 40.33 to 55.25 & 52.54 to 64.45 \\
\hline
\end{tabular}

It concludes from above highest change in temperature in blood is from 80.34 to $85.27 \%$ at frequency $2450 \mathrm{MHz}$ and change in temperature of bone is low as compared to other tissues when we move from 15 to $1 \mathrm{~cm}$ distance of mobile phone.

It is found that with the mobile phone radiations of above frequencies, the temperature of skin, blood, muscle and bone tissues slightly increases as the talking time is increased, but there is the highest raise in temperature in blood tissue and the lowest change in temperature in case of bone tissue. The rise in temperature of human body tissues is considerably large and the human body is not able to regulate it through the normal process of thermoregulation. Therefore human 
body may face thermal stress resulting into some diseases. When this temperature increases greater to $39^{\circ} \mathrm{C}$, it can produce many diseases in tissues and may become harmful for the life of tissues. Increase in temperature of these tissues cause thermal effect, which is harmful for human being.

\section{Acknowledgment}

The authors are grateful to the President Prof. Kamal Ghansala of Graphic Era University Dehradun who provides all the necessary facilities inside the University Campus.

\section{Author's Contributions}

All authors equally contributed in this work.

\section{Ethics}

This article is original and contains unpublished material. The corresponding author confirms that all of the other authors have read and approved the manuscript and no ethical issues involved.

\section{References}

Furse, C., D.A. Christensen and C.H. Durney, 2009. Basic Introduction to Bioelectromagnetics. 2nd Edn., CRC Press, ISBN-10: 1420055429, pp: 288.

Gabriel, C., S. Gabriel and E. Corthout, 1996a. The dielectric properties of biological tissues: I. Literature survey. Phys. Med. Biol., 41: 2231-2269. DOI: $10.1088 / 0031-9155 / 41 / 11 / 001$
Gabriel, S., R.W. Lau and C. Gabriel, 1996b. The dielectric properties of biological tissues: III. Parametric models for the dielectric spectrum of tissues. Phys. Med. Biol., 41: 2271-2293. DOI: $10.1088 / 0031-9155 / 41 / 11 / 003$

Gabriel, S., R.W. Lau and C. Gabriel, 1996c. The dielectric properties of biological tissues: II. Measurements in the frequency range $10 \mathrm{~Hz}$ to 20 GHz. Phys. Med. Biol., 41: 2251-2269. DOI: $10.1088 / 0031-9155 / 41 / 11 / 002$

Gandhi, F.M. and M.A.S. Mohammad, 2008. Dynamic source routing in adhoc wireless networks. J. Mobile Commun., 2: 39-45.

James, C.L., 2003. IEEE Antenas Propagat. Magaz., 45: 22-29.

Karunarathna, M.A. and I.J. Dayawansa, 2006. Energy absorption by the human body from RF and microwave emissions in Sri Lanka. Sri Lankan J. Phys., 7: 35-47. DOI: 10.4038/sljp.v7i0.207

Kumar, V., R.P. Vats and P.P. Pathak, 2008. Harmful effects of 41 and $202 \mathrm{MHz}$ radiations on some body parts and tissues. Ind. J. Biochem. Biophys., 45: 269- 274. PMID: 18788478

Osepchuk, J.M. and R.C. Petersen, 2003. Safety standards for exposure to electromagnetic fields. Bioelectromagneit. Suppl., 6: 7-16.

Ozen, S., S. Helnel and O. Cerezci, 2009. Heat analysis of biological tissue exposed to microwave by using thermal wave model of bio-heat transfer. J. Mobile Commun., 3: 445-449. 